\title{
Behavioral, Pharmacological, and Immunological Abnormalities after Streptococcal Exposure: A Novel Rat Model of Sydenham Chorea and Related Neuropsychiatric Disorders
}

\author{
Lior Brimberg', Itai Benhar2 ${ }^{2}$, Adita Mascaro-Blanco ${ }^{3}$, Kathy Alvarez ${ }^{3}$, Dafna Lotan', Christine Winter ${ }^{4,5}$, \\ Julia Klein ${ }^{5}$, Allon E Moses ${ }^{6}$, Finn E Somnier ${ }^{7}$, James F Leckman ${ }^{8}$, Susan E Swedo', \\ Madeleine W Cunningham ${ }^{*, 3,10}$ and Daphna Joel ${ }^{1,10}$
}

'School of Psychological Sciences, Tel Aviv University, Tel Aviv, Israel; ${ }^{2}$ Department of Molecular Microbiology and Biotechnology, Tel Aviv University, Tel Aviv, Israel; ${ }^{3}$ Department of Microbiology and Immunology, University of Oklahoma Health Sciences Center, OKlahoma City, OK, USA; ${ }^{4}$ Department of Psychiatry, Technical University Dresden, Dresden, Germany; ${ }^{5}$ Department of Psychiatry, Charité University Medicine Berlin, Berlin, Germany; ${ }^{6}$ Department of Clinical Microbiology and Infectious Diseases, Hadassah University Hospital, Jerusalem, Israel; ${ }^{7}$ Department of Clinical Biochemistry and Immunology, Statens Serum Institute, Copenhagen, Denmark; ${ }^{8}$ Yale Child Study Center, Departments of Pediatrics and Psychiatry, Yale University School of Medicine, New Haven, CT, USA; ${ }^{9}$ Pediatrics and Developmental Neuroscience Branch, National Institute of Mental Health, Bethesda, MD, USA

Group A streptococcal (GAS) infections and autoimmunity are associated with the onset of a spectrum of neuropsychiatric disorders in children, with the prototypical disorder being Sydenham chorea (SC). Our aim was to develop an animal model that resembled the behavioral, pharmacological, and immunological abnormalities of SC and other streptococcal-related neuropsychiatric disorders. Male Lewis rats exposed to GAS antigen exhibited motor symptoms (impaired food manipulation and beam walking) and compulsive behavior (increased induced-grooming). These symptoms were alleviated by the D2 blocker haloperidol and the selective serotonin reuptake inhibitor paroxetine, respectively, drugs that are used to treat motor symptoms and compulsions in streptococcal-related neuropsychiatric disorders. Streptococcal exposure resulted in antibody deposition in the striatum, thalamus, and frontal cortex, and concomitant alterations in dopamine and glutamate levels in cortex and basal ganglia, consistent with the known pathophysiology of SC and related neuropsychiatric disorders. Autoantibodies ( $\mathrm{lgG}$ ) of GAS rats reacted with tubulin and caused elevated calcium/calmodulindependent protein kinase II signaling in SK-N-SH neuronal cells, as previously found with sera from SC and related neuropsychiatric disorders. Our new animal model translates directly to human disease and led us to discover autoantibodies targeted against dopamine DI and D2 receptors in the rat model as well as in SC and other streptococcal-related neuropsychiatric disorders.

Neuropsychopharmacology (2012) 37, 2076-2087; doi:I0.1038/npp.2012.56; published online 25 April 2012

Keywords: streptococcus group A (GAS); Sydenham chorea (SC); PANDAS; dopamine; animal model; autoimmunity

\section{INTRODUCTION}

Infectious and immune factors are implicated in the pathogenesis of childhood neuropsychiatric disorders (Gordon, 2009; Murphy et al, 2006a), with the prototypical disorder being Sydenham chorea (SC). SC is induced by group A streptococcal (GAS) infection (Stollerman, 1997) and is characterized by involuntary movements and

*Correspondence: Professor MW Cunningham, Department of Microbiology and Immunology, Biomedical Research Center, University of Oklahoma Health Sciences Center, 975 NE I Oth Street, Oklahoma City, OK 73104, USA, Tel: + | 405 27| 3128, Fax: + I 405 27| 2217, E-mail: madeleine-cunningham@ouhsc.edu

${ }^{10} \mathrm{MWC}$ and DJ are co-senior authors.

Received 15 November 2011; revised 25 March 2012; accepted 26 March 2012 neuropsychiatric disturbances, including obsessive-compulsive symptoms and emotional lability (Marques-Dias et al, 1997; Swedo et al, 1989). It has been suggested that similar autoimmune mechanisms triggered by a GAS infection may lead to a syndrome termed PANDAS (pediatric autoimmune disorder associated with streptococcal infections) consisting of fine choreiform movements of fingers and toes as well as obsessive-compulsive symptoms or tics (Swedo et al, 1989). For reviews of the pros and cons of the PANDAS entity, see Swedo et al (2004) and Kurlan and Kaplan (2004), respectively. Over time, the term PANDAS has been applied to a very heterogeneous group of subjects with tics or obsessive-compulsive disorder (OCD) without fine choreiform movements and with other types of infections. In this study, PANDAS is defined as originally identified by Swedo et al (1998), and 
relates to a syndrome consisting of fine choreiform movements and obsessive-compulsive symptoms or tics that develop following exposure to GAS.

SC and PANDAS exhibit many clinical and laboratory similarities (for review, see Snider and Swedo, 2004), including association with antibodies against GAS and basal ganglia (Bronze and Dale, 1993; Church et al, 2003; Dale et al, 2006; Husby et al, 1976; Kirvan et al, 2003, 2006b; Morshed et al, 2001; Pavone et al, 2004); improvement after immunomodulatory therapies (Giedd et al, 1995, 1996, 2000); inflammation within the brain, particularly striatum and frontoparietal cortex, without evidence of neuronal degeneration (Colony and Malamud, 1956; Giedd et al, 1995); specific enlargement of basal ganglia during the active phase, which correlated with anti-streptococcal antibody titers (Giedd et al, 1995, 1996, 2000; Peterson et al, 2000); and induction of calcium/calmodulin-dependent kinase (CaMK) II signaling and dopamine release in catecholamine secreting SK-N-SH neuronal cell line by antibodies (Kirvan et al, 2003, 2006b). These findings implicate an immune response against the brain in the pathogenesis of SC and PANDAS (Snider and Swedo, 2004).

The aim of this study was to develop an animal model of streptococcal-induced behavioral changes resembling the SC/PANDAS disorder(s). We focused on four points. First, we tested whether exposure of the autoimmune-prone Lewis rat strain to GAS antigen leads to behavioral characteristics of SC/PANDAS, including impairments in fine motor control and gait, and increased activity, anxiety, and compulsivity (Gordon, 2009; Marques-Dias et al, 1997; Murphy et al, 2006b; Swedo et al, 1989). Second, we tested whether motor abnormalities and increased compulsivity in GAS rats would respond to the same drugs used to treat motor symptoms and compulsions in SC/PANDAS (D2 blockers and selective serotonin reuptake inhibitors (SSRIs), respectively, Demiroren et al, 2007; Shannon and Fenichel, 1990; Swedo et al, 1998; SE Swedo, unpublished observation). Third, we tested whether sera of GAS rats will have the same immune responses as sera from SC/PANDAS patients, including reaction against GAS antigens (Bronze and Dale, 1993; Husby et al, 1976; Kirvan et al, 2003, 2006b) and brain tissue and tubulin (Kirvan et al, 2007), and induction of CaMK II signaling in SK-N-SH neuronal cell line (Kirvan et al, 2003, 2006a). Fourth, we examined rat brains for antibody deposition (expected), gross neural damage (not expected), and changes in the dopaminergic, serotonergic, glutamatergic, and GABAergic systems, which have been implicated in the pathophysiology of motor and tic disorders and OCD (Bhattacharyya and Chakraborty, 2007; Singer and Loiselle, 2003; Singer and Minzer, 2003; Stein, 2002). Finally, because a D2 blocker was effective in reducing motor symptoms in GAS rats and dopamine content was increased in several regions in the brain of GAS rats, we tested whether sera IgG from GAS-exposed rats and humans with SC and PANDAS were reactive with human dopamine D1 and D2 membrane receptor antigens.

\section{MATERIALS AND METHODS}

\section{Animals}

Male Lewis rats (Harlen, Jerusalem, Israel), $\sim 5$ weeks old, were housed in groups of four to a cage under a reversed 12-h light-dark cycle (lights on at 1900-0700 h) with ad libitum food and water. A 22-h food restriction schedule with water freely available was initiated at age 9 weeks. All experimental protocols were carried out according to the guidelines of the institutional animal care and use committee of Tel-Aviv University.

\section{Human Sera}

Serum samples from SC and PANDAS patients and healthy controls were obtained from the National Institute of Mental Health (NIMH; Dr Swedo), the Child Study Center, Yale University (Dr Leckman), Statens Serum Institute in Copenhagen, Denmark (Dr Somnier), and the University of Oklahoma Health Sciences Center (Dr Cunningham). SC was diagnosed using criteria established by the Jones Criteria. PANDAS was diagnosed using the published NIMH criteria (Swedo et al, 1998), and included patients displaying fine piano playing movements of the fingers and toes in addition to obsessive-compulsive symptoms (Swedo et al, 1989). Attention deficit hyperactivity disorder was diagnosed on the basis of a structured psychiatric interview (K-SADS or DICA) and the Conners Parent and Teacher Rating Scales (Conners et al, 1998). Controls were group matched for age (ages 5-14 years), gender (male/ female: $\sim 4: 1$ ), socioeconomic status, and season of the year at entry into the study. Control subjects had normal physical examinations and were selected only if there was no lifetime personal history for the subject and any first-degree relative of a DSM-IV diagnosis of a chronic tic disorder, Tourette syndrome, OCD, or Attention deficit hyperactivity disorder. The anti-streptolysin $\mathrm{O}$ titer range for control subjects was 70-513, and for SC and PANDAS subjects it was 40-781. All human subjects gave informed consent and studies were approved by human subjects protection committees at the University of Oklahoma Health Sciences Center, Yale University, National Institute of Mental Health, and the Statens Serum Institute.

\section{Streptococcus pyogenes}

M protein type 18 (Manfraedo) was obtained from Dr Alon Moses (Hadassah University Medical Center, Jerusalem, Israel). Streptococci were grown as previously described (Fenderson et al, 1989). The cell pellets were stored frozen at $4{ }^{\circ} \mathrm{C}$ until used.

\section{Mutanolysin-Extracted GAS Antigen}

A whole cell digest of $M$ type $18 S$. pyogenes was prepared using a modification of a protocol described by Fenderson et al (1989) (see Supplementary Methods online).

\section{Immunization Protocol}

Rats were handled for $\sim 2$ min daily for 4 days before the beginning of the immunization protocol. Immunization was performed when the rats were 5 weeks of age. Before each injection, rats were lightly anaesthetized with Isoflorene (VetMarket, Petach Tikva, Israel). Each rat in the GAS group was immunized subcutaneously with $200 \mu$ l of $1: 1$ 
emulsion of phosphate-buffered saline (PBS) containing $1.2 \mathrm{mg}$ of the GAS antigen and Complete Freund's adjuvant (CFA, Sigma-Aldrich, Rehovot, Israel) supplemented with $4 \mathrm{mg}$ of heat-killed mycobacteria H37RA (Difco Laboratories, Detroit, MI) per ml similar to what was previously described for recombinant streptococcal $M$ protein-induced valvular heart disease (Quinn et al, 2001). Rats received in addition an intraperitoneal injection of $10^{10}$ heat-killed Bordetella pertussis (Bioport, Lansing, MI, USA) as an additional adjuvant. Heat-killed $B$. pertussis was added as it has previously been shown to increase the permeability of the blood brain barrier (Linthicum et al, 1982) and, indeed, in a pilot study in which we compared IgG deposits in the brain following immunization with and without B. pertussis, we found that omitting this bacteria from the immunization protocol almost completely abolished IgG immunostaining in the brain of GAS-exposed rats. After 14 and again after 28 days, each rat was boosted with $200 \mu 11: 1$ emulsion of incomplete Freund's adjuvant (IFA, Sigma-Aldrich): PBS containing $1.2 \mathrm{mg}$ of the GAS antigen. Control animals were immunized with PBS and adjuvants only.

\section{Behavioral Assessment}

Behavioral assessment was done in two experiments (see Table 1 for details). Manual ratings were performed in a blinded fashion.

Food manipulation. We assessed the ability of rats to manipulate a food pellet as previously described (Ayalon et al, 2004; also see Supplementary Methods online).

Food manipulation under haloperidol. Each rat in the haloperidol groups was injected with $0,0.05,0.1$, and $0.15 \mathrm{mg} / \mathrm{kg}$ haloperidol (the order of dose was randomly determined for each rat) $1 \mathrm{~h}$ before testing. Each rat in the vehicle groups was injected with vehicle $1 \mathrm{~h}$ before each of the 4 testing days.

Beam walking. Motor coordination and balance were assessed by measuring the ability of rats to traverse a wide $(5 \mathrm{~cm})$ and a narrow $(2.5 \mathrm{~cm})$ beam as previously

Table I Experimental Design

\begin{tabular}{|c|c|c|c|c|}
\hline \multirow[t]{2}{*}{ Cohort } & \multirow[t]{2}{*}{$\begin{array}{l}\text { Behavioral } \\
\text { assessment }\end{array}$} & \multicolumn{3}{|c|}{$\begin{array}{l}\text { Neuroanatomical and biochemical } \\
\text { assessment }\end{array}$} \\
\hline & & $\begin{array}{l}\text { Staining } \\
\text { for IgG } \\
\text { deposits }\end{array}$ & $\begin{array}{l}\text { Histological } \\
\text { assessment }\end{array}$ & $\begin{array}{l}\text { Biochemical } \\
\text { assessment }\end{array}$ \\
\hline I & $\begin{array}{l}\text { Activity; plus maze; } \\
\text { induced-grooming } \\
\text { under vehicle or } \\
\text { paroxetine; food } \\
\text { manipulation }\end{array}$ & $\begin{array}{l}21 \text { GAS rats }^{\mathrm{a}} \\
\text { 17 control } \\
\text { rats }^{\mathrm{a}}\end{array}$ & All rats & \\
\hline 2 & $\begin{array}{l}\text { Food manipulation } \\
\text { under vehicle or } \\
\text { haloperidol; beam } \\
\text { walking }\end{array}$ & $\begin{array}{l}14 \text { GAS rats } \\
17 \text { control rats }\end{array}$ & & $\begin{array}{l}7 \text { GAS rats }^{\mathrm{b}} \\
6 \text { control rats }^{\mathrm{b}}\end{array}$ \\
\hline
\end{tabular}

aSections of 14 and $40 \mu \mathrm{m}$ were taken alternately from the same animals for the detection of IgG deposits and histology, respectively.

${ }^{\mathrm{b}}$ These rats were not administered haloperidol. described (Urakawa et al, 2007; also see Supplementary Methods online).

Activity test. Rats were individually placed in an activity box and allowed $30 \mathrm{~min}$ of free exploration during which their activity level was assessed, as previously described (Piontkewitz et al, 2010; also see Supplementary Methods online).

Grooming. The assessment of induced-grooming followed that of Greer and Capecchi (2002) (also see Supplementary Methods online).

Grooming under paroxetine. Paroxetine $(9.25 \mathrm{mg} / \mathrm{kg})$ or vehicle was administered $30 \mathrm{~min}$ before each of the three grooming sessions.

Elevated plus maze. The anxiety-like behavior of rats was assessed in the elevated plus maze paradigm as described previously (Hogg, 1996; also see Supplementary Methods online).

\section{Drugs}

Haloperidol (VetMarket) and paroxetine (Unipharm, Ramat-Gan, Israel) were prepared as previously described (Brimberg et al, 2007; Joel and Doljansky, 2003), and injected i.p. 60 and $30 \mathrm{~min}$, respectively, before behavioral assessment. No-drug controls received an equivalent volume of the corresponding vehicle (saline and double distilled water, respectively).

\section{Immunological Assessment}

Immunological assessment was done on animals from the two cohorts of behavioral experiments (see Table 1 for details).

For reagents, ELISAs, and western blots, see Supplementary Methods online.

CaMK II assay. We assessed CaMK II activity as previously described (Kirvan et al, 2003; also see Supplementary Methods online). IgG inhibition was performed by adding either anti-rat IgG agarose beads (Sigma, St Louis, MO) or control bovine serum albumin-agarose beads (Sigma) to experimental (GAS) sera (see Supplementary Methods online).

\section{Neuroanatomical and Biochemical Assessment}

Neuroanatomical and biochemical assessment was done on animals from the two cohorts of behavioral experiments (see Table 1 for details).

\section{Neuroanatomical Assessment.}

Preparation of tissue sections: Brains were obtained and prepared as previously described (Klavir et al, 2009; see also Supplementary Methods online).

Immunostaining for IgG deposits: To assess IgG deposits in the brain, $14 \mu \mathrm{m}$ sections were incubated in biotinylated secondary anti-rat IgG (see Supplementary Methods online).

Histology: To assess cellular infiltrations and possible neuronal loss, $40 \mu \mathrm{m}$ sections were stained with Thionin blue. All slides were coded for blinded analysis. 
Biochemical Assessment. We assessed the levels of the monoamines dopamine (as well as its metabolites 3,4dihydroxyphenylacetic acid (DOPAC) and homovanillic acid (HVA), and dopamine turnover) and serotonin (5hydroxytryptamine (5-HT) and its metabolite, 5-hydroxy indole acetic acid (5-HIAA)) as well as glutamate and GABA $(\gamma$-amino butyric acid) in tissue samples from the caudateputamen, nucleus accumbens, globus pallidus, entopeduncular nucleus, substantia nigra, ventral tegmental area, medial frontal cortex, orbitofrontal cortex, the dorsomedial nucleus of the thalamus, and the subthalamic nucleus as previously described (Klavir et al, 2009; see also Supplementary Methods online).

\section{Statistical Analysis}

We used two-sample Student's $t$-tests to compare means of two independent groups. When more groups were compared, we used analysis of variance (ANOVA; the specific factors for each analysis are given in the Results section) together with planned comparisons between GAS and control rats to reveal the effect of immunization (the specific comparisons are detailed in the Results section). In procedures where a drug was administered, we additionally compared the effects between drug- and vehicle-treated rats within each immunization condition. Human samples were statistically analyzed using the Mann-Whitney test. We considered values as significant when $P<0.05$. When multiple comparisons were done, the Keppel multiple comparisons correction was used. All data are presented as means \pm SEM.

\section{RESULTS}

\section{Motor and Behavioral Abnormalities Following Streptococcal Exposure}

Figure 1 presents food manipulation scores (Figure 1a), time to traverse a beam (Figure 1b), time spent grooming in the induced-grooming assay (Figure 1c), and activity scores in automated activity chambers (Figure 1d) of GAS-exposed rats and adjuvant control rats. As can be seen, GAS rats were impaired in manipulating food (Figure $1 \mathrm{a}, \mathrm{t}(33)=3.45$, $P<0.005)$ and in traversing the narrow $(2.5 \mathrm{~cm})$ but not the wide $(5.0 \mathrm{~cm})$ beam, as evident in a longer time to traverse the beam (Figure 1b; Immunization $\times$ Beam Width mixed ANOVA: Immunization, $\mathrm{F}(1,17)=1.65, \quad P=0.21 ; \quad$ Beam Width, $\mathrm{F}(1,17)=7.8, P<0.05$, Immunization $\times$ Beam Width interaction, $\mathrm{F}(1,17)=4.48, P<0.05)$. Planned comparisons between control and GAS groups within each beam width demonstrated that GAS rats were impaired only on the $2.5 \mathrm{~cm}$ beam $(P=0.02)$; there were no differences between the groups in the number of foot slips (data not shown, all $P$-values $>0.4)$. GAS rats spent more time grooming on the third day of testing in the induced-grooming assay (Figure 1c; Immunization $\times$ Session mixed ANOVA: Immunization, $\mathrm{F}(1,14)=10.3, P<0.01$, Session, $\mathrm{F}(2,28)=$ 1.63, $P=0.21$, Immunization $\times$ Session interaction, $\mathrm{F}(2,28)=$ 3.75, $P<0.05)$. Planned comparisons between GAS and control groups within each session revealed a significant effect on the third block only $(P<0.05)$. Importantly, GASexposed rats did not have any gross indications of skin lesions, nor did they show loss of hair, which could indicate an increase in spontaneous grooming. GAS rats tended to be

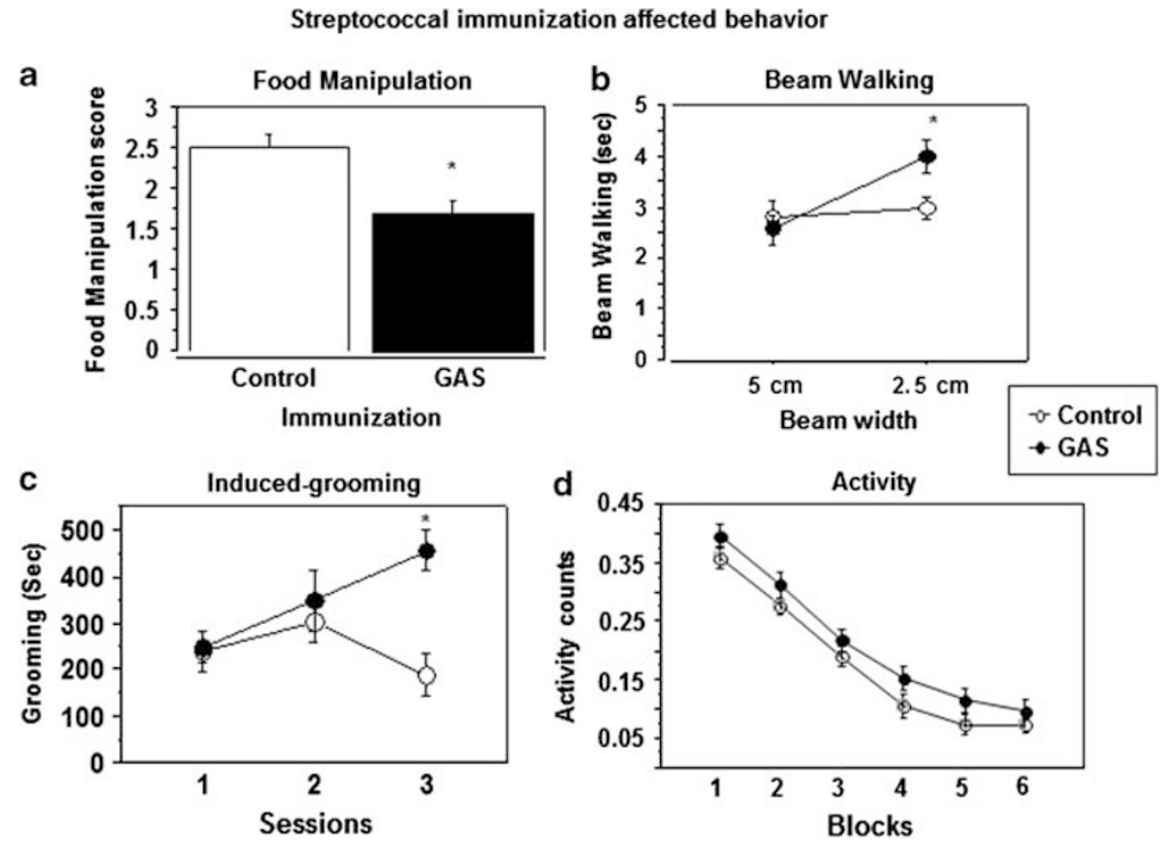

Figure I Effects of streptococcal exposure on (a) food manipulation, (b) beam walking, (c) grooming, and (d) activity. (a) Food manipulation was impaired in GAS-exposed rats. The mean and SE of food manipulation score of GAS rats $(n=19)$ and control rats $(n=16)$. (b) Beam walking was impaired in GASexposed rats on the narrow beam only. The mean and SE of the time spent on the wide and narrow beams of GAS rats $(n=9)$ and control rats $(n=10)$. (c) Induced-grooming was enhanced by GAS exposure. The mean and SE of the duration of induced-grooming on three sessions of GAS ( $n=8)$ and control $(n=8)$ rats. (d) Activity tended to increase following GAS exposure. The mean and SE of activity counts, in 5 min blocks, of GAS ( $n=20)$ and control $(n=17)$ rats during a 30-min activity session. *Significantly different from the control group, $P<0.05$. 
Treatment of immunized rats with haloperidol or paroxetine abrogated behavioral changes
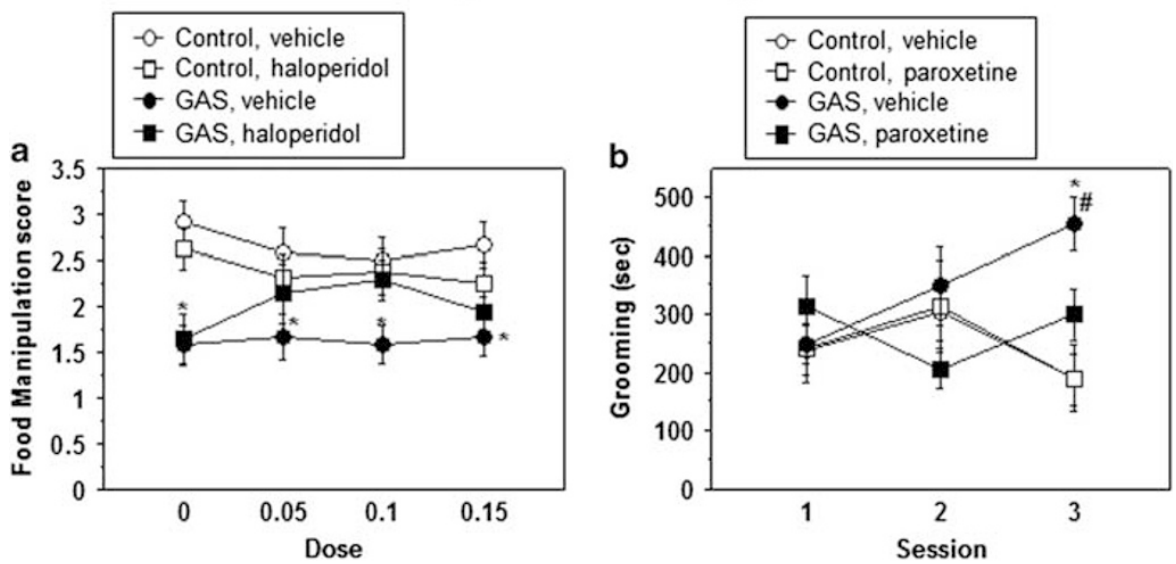

Figure 2 Effect of (a) haloperidol on food manipulation and (b) paroxetine on grooming. GAS-treated rats were impaired in manipulating food and haloperidol ameliorated this impairment, whereas paroxetine normalized the duration of induced-grooming. (a) Following a habituation session and a baseline session, rats underwent four additional food manipulation sessions separated by 3 wash-out days. Each rat in the haloperidol groups was injected with $0,0.05,0.1$, or $0.15 \mathrm{mg} / \mathrm{kg}$ I h before testing. Each rat in the vehicle groups was injected with vehicle I h before testing on each of the 4 testing days. The figure presents the mean and SE of food manipulation scores of GAS $(n=7)$ and control $(n=8)$ rats treated with haloperidol, and of GAS $(n=6)$ and control $(n=6)$ rats treated with vehicle across all sessions. (b) The mean and SE of the duration of grooming during the three grooming sessions of GAS and control rats treated with $9.25 \mathrm{mg} / \mathrm{kg}$ paroxetine or vehicle $30 \mathrm{~min}$ before each session (n per group: control-vehicle $=8$, GAS-vehicle $=8$, controlparoxetine =6, GAS-paroxetine = I0; note that the vehicle groups are the same as those appearing in Figure Ic). *Significantly different from the controlvehicle group, $P<0.05$; "Significantly different from GAS-drug group, $P<0.005$.

more active than control rats throughout the 30 min activity session (Figure 1d; Immunization $\times$ Block mixed ANOVA: Immunization, $\mathrm{F}(1,35)=2.76 P=0.1$, Blocks, $\mathrm{F}(5,175)=$ $187.56, \quad P<0.0001$, Immunization $\times$ Blocks interaction, $\mathrm{F}(5,175)=0.25, P=0.93)$, but did not differ significantly from controls on the elevated plus maze (data not shown, $P=0.7)$.

\section{Amelioration of Motor Symptoms by the Dopamine D2 Receptor Antagonist Haloperidol and Compulsive Behavior by the SSRI Paroxetine}

Figure 2 shows food manipulation scores following treatment with different doses of haloperidol $(0.05,0.1$, and $0.15 \mathrm{mg} / \mathrm{kg}$, Figure 2a) and time spent grooming in the induced-grooming assay following administration of $9.25 \mathrm{mg} / \mathrm{kg}$ paroxetine (Figure 2b) of GAS and control rats. As can be seen, haloperidol dose-dependently restored the ability of GAS rats to manipulate food, except for the highest dose of this drug, which tended to impair performance in GAS and control rats (Figure 2a; Immunization $\times$ Drug $\times$ Dose mixed ANOVA: Immunization, $\mathrm{F}(1,23)=12.6, P<0.005$, Drug, $\mathrm{F}(1,23)=0.6$, $P=0.8$, Dose, $\mathrm{F}(3,69)=0.13, P=0.9$, Immunization $\times$ Drug interaction, $\mathrm{F}(1,23)=2.6, \quad P=0.12$, Immunization $\times$ Dose interaction, $\mathrm{F}(3,69)=3.48, P<0.05$, Drug $\times$ Dose interaction, $\mathrm{F}(3,69)=1.3, P=0.275$, Immunization $\times$ Drug $\times$ Dose interaction, $\mathrm{F}(3,69)=0.41, P=0.74)$. Planned comparisons between control and GAS groups within the vehicle condition and between GAS rats treated with haloperidol and control rats treated with $0 \mathrm{mg} / \mathrm{kg}$ haloperidol confirmed that GAS rats were impaired in manipulating food, and that 0.05 and $0.1 \mathrm{mg} / \mathrm{kg}$ haloperidol reversed this impairment (all $P$-values $<0.05)$. Administration of the SSRI paroxetine decreased induced-grooming in GAS rats without affecting inducedgrooming in control rats (Figure 2b; Immunization $\times$
Drug $\times$ Session mixed ANOVA: Immunization, $F(1,28)$ $=5.29 P<0.05$, Drug, $\mathrm{F}(1,28)=1.56, P=0.22$, Session, $\mathrm{F}(2,56)$ $=0.4, P=0.66$, Immunization $\times$ Drug interaction, $\mathrm{F}(1,28)=$ 2.1, $P=0.15$, Immunization $\times$ Session interaction, $\mathrm{F}(2,56)=$ 4.65, $P<0.05, \quad$ Drug $\times$ Session interaction, $F(2,56)=1.3$, $P=0.25$, Immunization $\times$ Drug $\times$ Session interaction, $\mathrm{F}(2,56)=$ $1.4, P=0.23)$. Planned comparisons revealed that the GASvehicle group was significantly different from the controlvehicle and GAS-paroxetine groups, all $P$-values $<0.05$.

\section{GAS Rat Sera Reacted with Streptococcal and Brain Antigens}

As expected, IgG antibodies in GAS rat sera were significantly (all $P$-values $<0.0001$ ) elevated against the GAS cell wall antigen, the immunogen, as well as against peptidoglycan-polysaccharide (PG-PS) and $N$-acetyl- $\beta$-Dglucosamine (GlcNAc), the dominant epitope of GAS carbohydrate (see Supplementary Material, Figure S1). As shown in humans with SC (Kirvan et al, 2007), GAS rat sera IgG reacted with brain-derived tubulin (see Supplementary Material, Figure S2).

\section{Antibody-Mediated Neuronal Cell Signaling Was Elevated in GAS Rat Sera and Signaling Activity Was Depleted by Anti-IgG}

Sera of GAS rats activated CaMK II signaling in a catecholamine-secreting neuronal cell line (SK-N-SH) to a level approximately three times higher than sera from control rats (Figure $3 \mathrm{a} ; \mathrm{t}(16)=3.66, P<0.005$ ). In GAS rats, CaMK II activation was reduced by $60 \%$ after a single absorption of serum IgG by anti-IgG beads, whereas bovine serum albumin (BSA) beads did not significantly remove CaMK II activity from the sera (Figure 3b). These data demonstrate that CaMK II activation was through an 
IgG antibodies from streptococcal immunized rats induced CaM kinase II

a

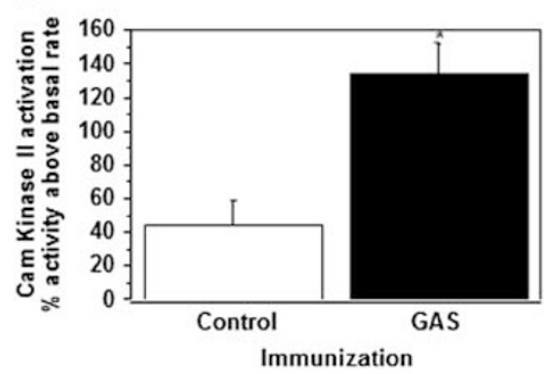

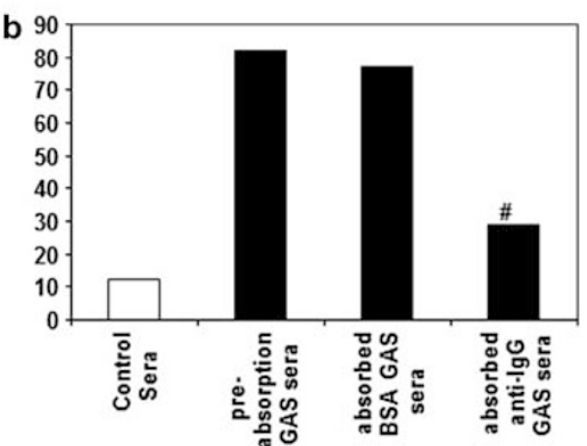

Streptococcal immunization induced antibodies against D1 and D2 Dopamine receptors

C

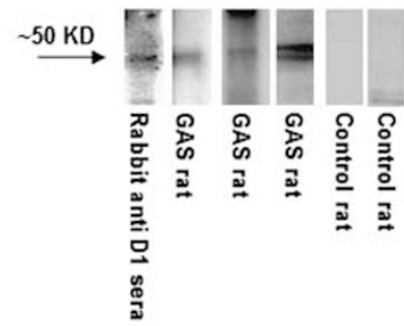

d

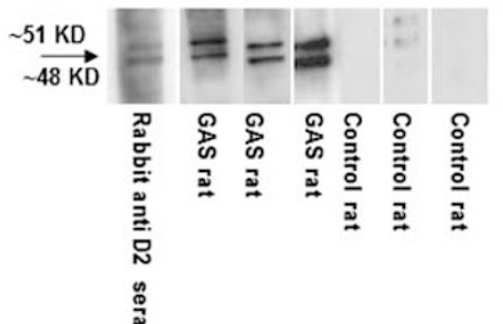

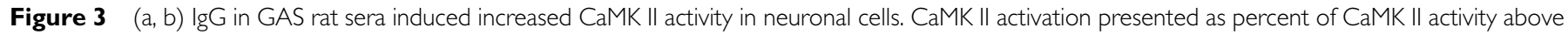

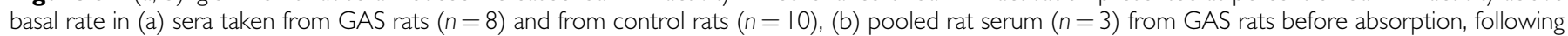

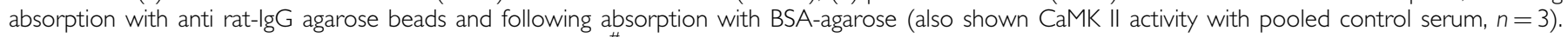

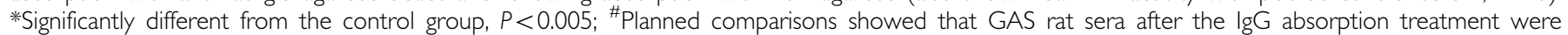

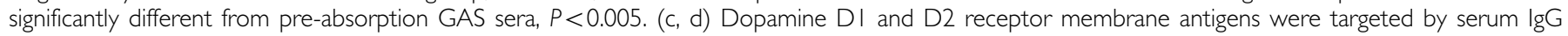

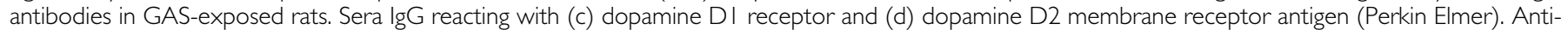

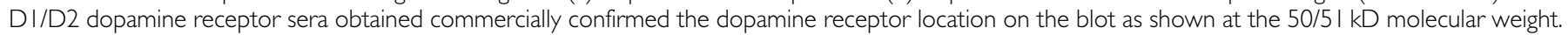

antibody IgG-mediated signaling mechanism as previously reported in SC and PANDAS (Kirvan et al, 2007).

\section{IgG Deposited in Striatum, Thalamus, and Frontal Cortex in GAS Rats}

Intense IgG deposition was observed in the striatum, thalamus, and frontal cortex of GAS-exposed rats, but not in their hippocampus and cerebellum, whereas little or no IgG staining was seen in the brains of control rats (Figure $4 \mathrm{a}-\mathrm{e}$ ). In GAS rats, anti-IgG immunolabeling within the striatum, thalamus, and frontal cortex appeared to label neuronal cells more intensely (Figure 4a-e). Thionin blue staining did not reveal evidence of gross neuronal damage (Figure 4a-e). All secondary antibody controls were negative for all tissues from the GAS-exposed and the control rats (data not shown).

\section{Streptococcal Exposure Increased Levels of Dopamine} and Decreased Levels of Glutamate in the Brains of Immunized Rats

HPLC analysis of the brains of GAS and control rats revealed increased dopamine $(P=0.0049)$ and decreased glutamate $(P=0.0012)$ in the medial frontal cortex of GAS rats (see Table 2 for significant results and Supplementary Material, Table S1 for the full analysis). Dopamine was also increased in the entopeduncular nucleus $(P=0.0058$; the rat analog of the primate internal segment of the globus pallidus) of GAS rats. In addition, the dopamine metabolite HVA tended to be lower in the medial frontal cortex $(P=0.0147)$, entopeduncular nucleus $(P=0.0187)$, and orbitofrontal cortex $(P=0.0233)$ of GAS rats compared with controls. Dopamine turnover was not significantly affected by GAS exposure (see Supplementary Material, Table S1).

\section{GAS Rat Sera IgG Targeted Human Dopamine D1 and D2 Receptor Membrane Antigens}

Because dopamine D2 receptor blockers are used to treat chorea in SC and motor symptoms in PANDAS, and CaMK II signaling may lead to dopamine release, we investigated rat sera for IgG reactive with the human dopamine D1 and $\mathrm{D} 2$ receptor membrane antigens obtained from $\mathrm{D} 1 \mathrm{R}$ and D2R stably transfected cells. Sera from GAS rats reacted significantly more with human D1 and D2 dopamine receptor membrane antigens compared with control rats (ELISA: D1, control rat sera $(n=10)$ mean titer $=328.0$ $(\mathrm{SE}=42.1)$, GAS rat sera $(n=11)$ mean titer: 640.0 $(\mathrm{SE}=105.7), \mathrm{t}(19)=2.6, \quad P<0.05 ; \mathrm{D} 2$, control rat sera $(n=16)$ mean titer $=960.0 \quad(\mathrm{SE}=82.6)$, GAS rat sera $(n=18) \quad$ mean titer: $1280.0 \quad(\mathrm{SE}=126.7), \mathrm{t}(32)=2.0$, $P<0.05)$. Reactivity of the GAS rat sera with the D1 and D2 dopamine receptor antigen was confirmed in the western immunoblot (Figure $3 \mathrm{c}$ and d). Anti-streptococcal rat sera $(\mathrm{IgG})$ reacted with human dopamine receptors D1 and D2 (protein bands at 50/51 kD) that were identified in 


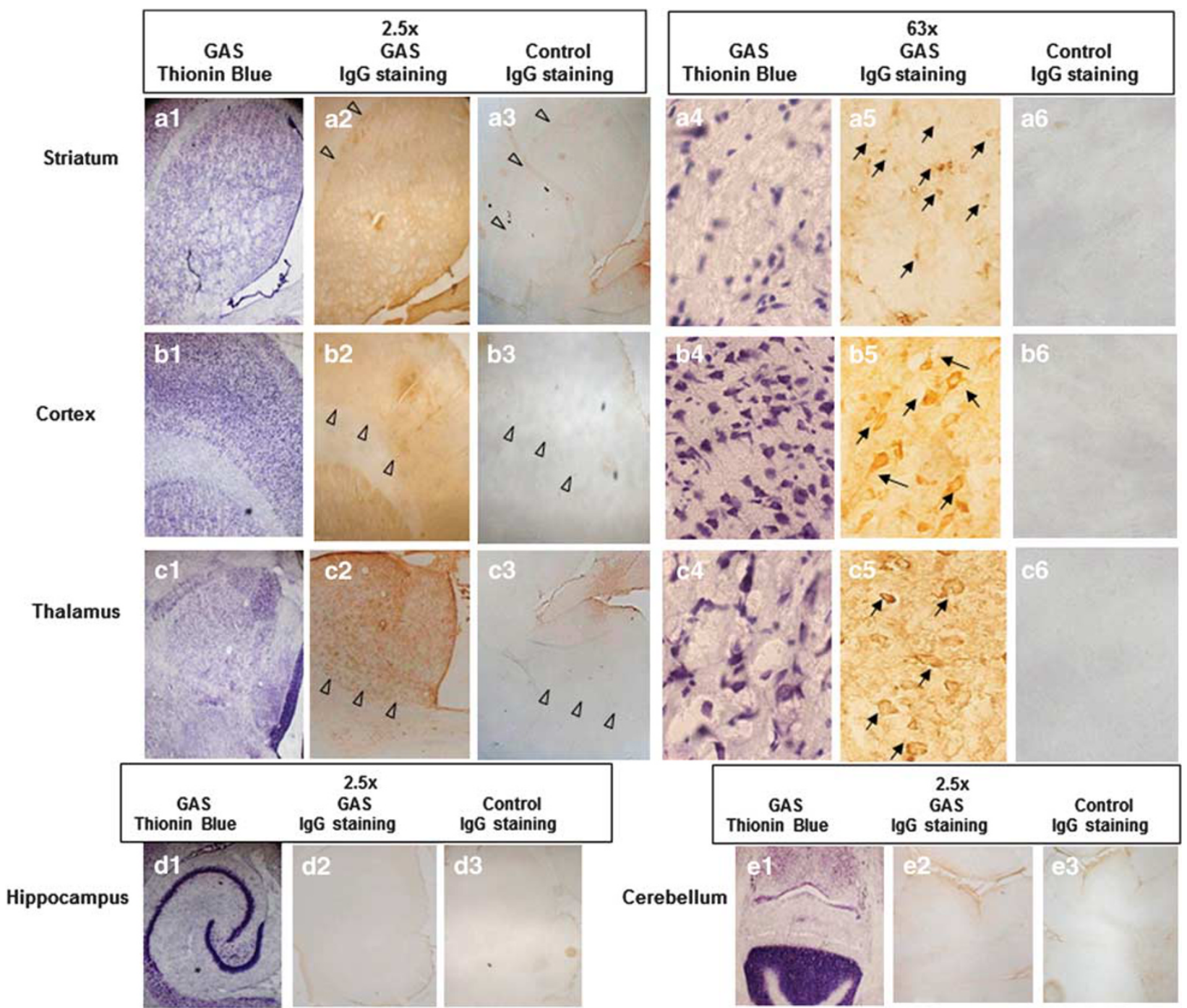

Figure 4 IgG deposition in brains of GAS-exposed rats. Thionin blue stained cell structures in transversal tissue sections taken through the striatum (a I, 4), cortex (bl, 4), thalamus ( $\mathrm{cl}, 4)$, hippocampus $(\mathrm{dl})$, and cerebellum (el) of a GAS-exposed rat at a magnification of $\times 2.5(\mathrm{I})$ and $\times 63(4)$. IgG deposits in brain sections taken at the same level as $(\mathrm{I})$ from a GAS $(2,5)$ and a control rat $(3,6)$ at a magnification of $\times 2.5(2,3)$ and of $\times 63(5,6)$. Tissue sections were incubated in biotinylated-anti-rat IgG and then incubated with avidin using Vectastain ABC kit (Vector Laboratories, Burlingame, CA, USA). Anti-lgG binding to brain sections was detected using diaminobenzidine for visualization of antibody deposition. Arrowheads illustrate the margins of regions of interest. Arrows mark staining of neuronal cells.

Table 2 Increased Dopamine in Medial Prefrontal Cortex and Entopeduncular Nucleus in GAS-Exposed Rats ${ }^{2}$

\begin{tabular}{|c|c|c|c|c|c|c|}
\hline \multirow[t]{2}{*}{ Transmitter ( $\mu$ mol/g protein) } & \multicolumn{2}{|c|}{ Control } & \multicolumn{2}{|c|}{ GAS } & \multirow[t]{2}{*}{ t-Value } & \multirow[t]{2}{*}{ Significance } \\
\hline & Average & SE & Average & SE & & \\
\hline \multicolumn{7}{|l|}{ Medial prefrontal cortex (CGI) } \\
\hline Glutamate & 131.58 & 3.38 & 109.6 & 5.1 & 3.589 & 0.0049 \\
\hline Dopamine & 3.487 & 0.506 & 6.548 & 0.458 & -4.387 & 0.0012 \\
\hline \multicolumn{7}{|c|}{ Entopeduncular nucleus (globus pallidus internal) } \\
\hline Dopamine & 10.219 & 0.997 & 16.08 & 1.34 & -3.497 & 0.0058 \\
\hline
\end{tabular}

${ }^{a}$ Determined by post-mortem high-performance liquid chromatography (HPLC).

the blot as the correct size of $50 \mathrm{kD}$ with rabbit anti-D1 and 48 and $51 \mathrm{kD}$ anti-D2 dopamine receptor sera. No reactivity was observed in the western blot for control rat sera.
To compare the rat model with human disease, reactivity of human serum samples obtained from children who were acutely ill with SC or PANDAS is shown in Figure 5. Sera 
Antibodies (lgG) against Dopamine Receptors D1 and D2 in SC and PANDAS
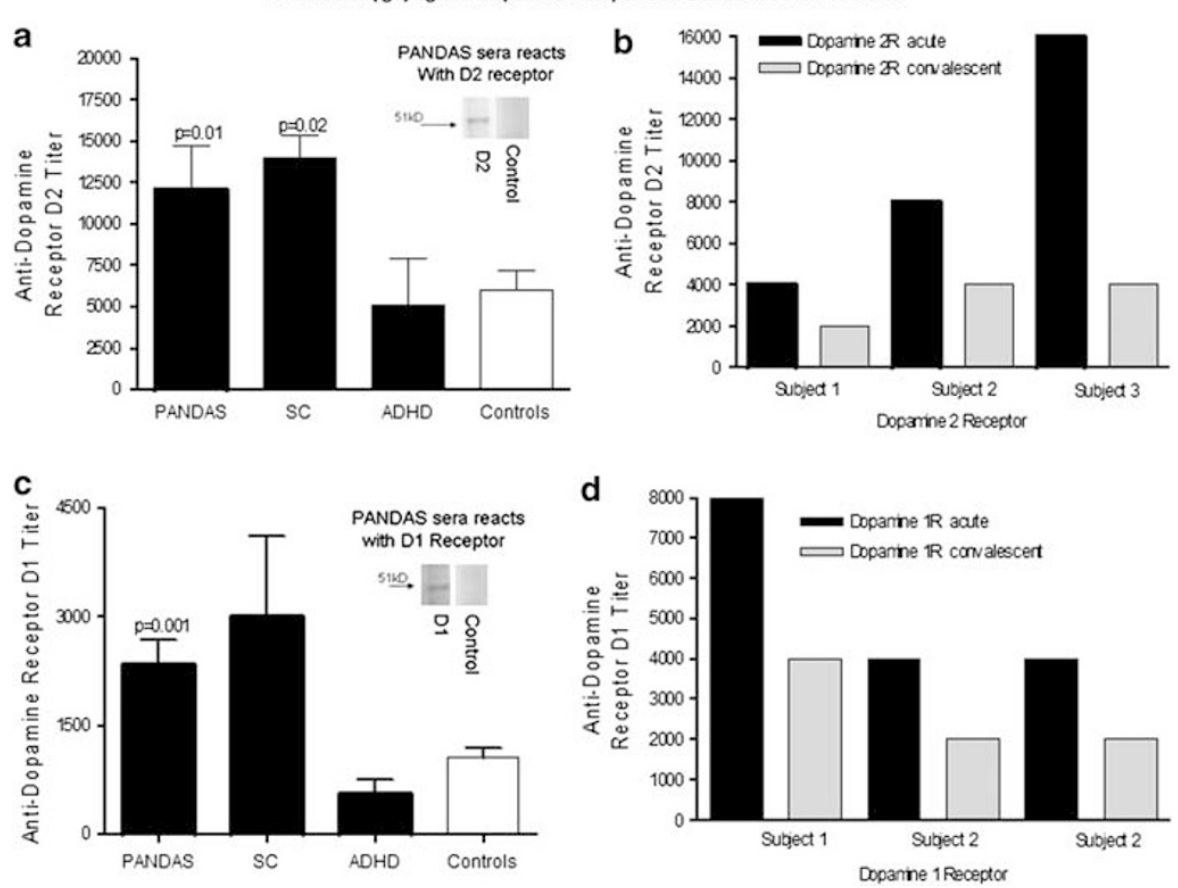

Figure 5 Anti-dopamine DI and D2 receptor membrane antigen antibodies in SC and PANDAS. (a, c) Antibodies (lgG) in sera from SC ( $n=8)$ and PANDAS $(n=27)$, but not attention deficit hyperactivity disorder (ADHD, $n=5)$ or healthy controls $(n=19)$, were significantly elevated against dopamine DI (a) and D2 (c) receptor membrane antigens. Inset of a western blot in the graphs in (a) and (c) confirm lgG reactivity of PANDAS sera with purified human DI and D2 dopamine receptor membrane antigen $(50 / 5 \mathrm{l} \mathrm{kD})$ but not lgG in sera from healthy controls as shown as negative in the western blot. PANDAS sera used in the western blots had elevated antibody titers against DI/D2 membrane receptors ( $\geqslant 8000)$ in the ELISA, whereas healthy controls had lower titers of antibody for DI (500) or for D2 (2000) receptors. (b, d) Antibodies (lgG) in sera from three PANDAS patients were elevated against dopamine DI (b) and D2 (d) receptor membrane antigens during active symptoms and were decreased during convalescence.

IgG from SC reacted significantly more with the dopamine D2 receptor membrane antigen compared with healthy controls $(P<0.01)$, whereas the PANDAS sera reacted significantly more than the controls with both the D1 $(P=0.001)$ and D2 $(P=0.02)$ receptor membrane antigens (Figure $5 \mathrm{a}$ and $\mathrm{c}$ ). In addition, the level of anti-D2 and antiD1 membrane antigen IgG was lower in convalescence than during active acute symptoms in the three patients for whom we had sera from these two time points (Figure $5 \mathrm{~b}$ and d). Human sera IgG from PANDAS sera reacted with a $50-\mathrm{kD}$ band in both the D1R/D2R membrane preparations comparable to the anti-D1R/D2R rabbit sera control (see inset of the blot in the graph showing the ELISA results of the different groups).

\section{DISCUSSION}

There have been previous attempts to develop an animal model of streptococcal-related neuropsychiatric disorders. The first line of studies infused the serum of patients into the striatum of rats. Although early studies reported that this manipulation led to the emergence of stereotypic behaviors (Hallett et al, 2000; Taylor et al, 2002), later studies have failed to replicate this effect (Loiselle et al, 2004; Singer et al, 2005). A different approach was taken by Hoffman et al (2004). Female mice were immunized and boosted with a GAS (type M6) antigen in Freund's adjuvant. A subset of these mice developed motoric and behavioral disturbances that were correlated with immu- noreactivity to the deep cerebellar nuclei (Hoffman et al, 2004). In a subsequent study, passive transfer of antistreptococcal sera from immunized mice to naive mice (concomitant with lipopolysaccharides (LPS) to break the blood brain barrier) resulted in antibody deposition in the hippocampus and in the development of motoric and behavioral disturbances similar to those seen in immunized mice (Yaddanapudi et al, 2009). Both studies (Hoffman et al, 2004; Yaddanapudi et al, 2009) support a role for antibodies in the induction of behavioral alterations. Although the mouse model was successful in showing behavioral changes in streptococcal-immunized mice and was related to passively transferred antibody, it did not investigate specific neural and immune characteristics reported previously in SC and PANDAS (Husby et al, 1976; Kirvan et al, 2003, 2006a).

Our new model demonstrated that exposure of male Lewis rats to GAS antigens leads to a syndrome that resembles behavioral, pharmacological, immunological, and neural characteristics of SC and PANDAS (as originally identified by Swedo et al, 1998). Behaviorally, streptococcalexposed rats were impaired in manipulating food and in traversing a narrow, but not a wide, beam. The finding that GAS-exposed rats were not impaired in traversing the wide beam suggests that they did not have reduced motivation to reach the home cage, nor some other nonspecific impairment that resulted in general slowness. These conclusions are further supported by our observation that there were no differences between GAS-exposed and control rats in the acquisition of lever pressing for a food reward (Brimberg 
and Joel, unpublished observation). Taken together, the pattern of results obtained suggests that streptococcal immunization resulted in specific motor impairments in fine motor control and gait, as previously reported in SC and PANDAS (Gordon, 2009), without affecting motivation and gross motor control. Streptococcal-exposed rats also showed increased grooming in the induced-grooming assay, with no signs of skin lesions or hair loss, which could indicate an increase in spontaneous grooming. These findings suggest that the increase in induced-grooming in GAS-exposed rats represents a form of compulsive responding, consistent with reports of obsessive and compulsive symptoms in SC and PANDAS (Gordon, 2009; Murphy et al, 2006a, b; Swedo et al, 1989). The relevance of impaired food manipulation and increased induced-grooming in GAS-exposed rats to SC and PANDAS was supported by demonstrating that these symptoms were alleviated by administration of haloperidol and paroxetine, respectively, which are used to treat motor and compulsive symptoms in SC and PANDAS (Demiroren et al, 2007; Shannon and Fenichel, 1990). (Paroxetine was administered in the present experiment for 3 days, whereas in OCD patients SSRIs are effective only after several weeks of repeated administration. However, several authors have pointed to some difficulties with the notion of delayed drug effects in psychiatric disorders, and there is an ongoing debate regarding the implications of demonstrating beneficial effects after acute drug administration to the validity of animal models (for review and references, see Albelda and Joel, 2012). Although this issue is relevant for animal models of many psychiatric disorders (in which response to pharmacological treatment is evident only after several weeks of treatment), whether emphasis is placed on the treatment regimen greatly depends on the psychopathology that is being modeled. Among animal models of OCD, some models used chronic (3-5 weeks of daily injections) or subchronic (3 injections over $24 \mathrm{~h}$ ) regimen of drug administration to establish predictive validity, whereas others have mainly used acute administration (for a recent review, see Albelda and Joel, 2012). In view of the above, we think that the present finding that subchronic administration of paroxetine decreased induced-grooming in GAS-exposed rats strengthens the validity of our model.)

Immunologically, exposure to GAS led to induction of antibrain and anti-tubulin antibodies, as well as antibodies that activated CaMK II signaling, as has previously been found for IgG in sera obtained from SC and PANDAS (Kirvan et al, 2007; Kirvan et al, 2003, 2006a). Moreover, our study demonstrated the role of antibodies in neuronal cell signaling, as removal of IgG diminished neuronal cell signaling of GAS rat sera. Finally, antibodies (IgG) deposited in the striatum, thalamus, and frontal cortex of streptococcal-exposed rats, in line with evidence implicating the striatum in the pathogenesis of SC and PANDAS, and the striatum and frontal cortex in the pathophysiology of OCD. Thus, anti-striatal antibodies have been reported in serum and cerebrospinal fluid of SC (Bronze and Dale, 1993; Church et al, 2002; Husby et al, 1976), PANDAS (Gause et al, 2009; Pavone et al, 2004), and adult OCD patients (Bhattacharyya et al, 2009). In addition, functional imaging studies found hypermetabolism in the striatum of SC patients (Dilenge et al, 1999; Weindl et al, 1993 ) and in the striatum and frontal cortex of OCD patients
(Huyser et al, 2009), which normalized after successful treatment (Dilenge et al, 1999; Huyser et al, 2009; Weindl et al, 1993), and imaging studies in PANDAS patients reported that the basal ganglia were enlarged (Giedd et al, 2000) and size correlated with anti-neural antibody titers (Giedd et al, 2000; Peterson et al, 2000).

Our results further support a causal relationship between exposure to GAS and the development of anti-brain antibodies and behavioral disorders, as previously demonstrated by Hoffman et al (2004). The difference in the targets of autoantibodies between the study of Hoffman et al (2004) and the present study may be because of differences in the animal species (mice $v s$ rats) or sex (female $v s$ male) used, in the specific GAS strain and antigen (M6 vs M18) used for immunization, or in the means used to disrupt the blood brain barrier (no substance other than Freund's adjuvants $v s$ the addition of $B$. pertussis).

Our study is the first to show the relevance of streptococcal-induced behavioral disorders in rats to the human condition by demonstrating a similar pharmacological profile and a similar immunological response phenotype. In addition to translation of behavioral and immunological effects of exposure to GAS in our rat model to humans, our study revealed that exposure to GAS resulted in alteration in the functioning of the glutamatergic and dopaminergic systems. Specifically, compared with control rats, streptococcal-exposed rats exhibited lower glutamate content in the medial frontal cortex and an increase in dopamine in this region and in the entopeduncular nucleus. In the case of glutamate, the finding is of special interest as there are reports of reduced anterior cingulate glutamate concentration in pediatric OCD (Rosenberg et al, 2004). In addition, recent studies have shown that glutamatergic agonists can ameliorate symptoms in OCD (Kushner et al, 2007), and it has been suggested that glutamatergic agents can be useful in treating Tourette's syndrome (Singer et al, 2009). As the effects of glutamatergic agents have not been tested in the present study, we cannot determine whether the alterations in the glutamatergic system played a causal role in the induction of symptoms in the model.

However, in the case of dopamine, several lines of evidence suggest a causal role for dopaminergic alterations in the induction of symptoms in the model. Specifically, changes in dopaminergic function in the medial frontal cortex have previously been shown to correlate with impaired forelimb movement and beam walking and with excessive grooming (Homberg et al, 2002; Urakawa et al, 2007). Increased dopaminergic function has been implicated in the pathophysiology of chorea (for review, see Gordon, 2009), and a decrease in plasma levels of catecholamine metabolites has been found in SC patients (Umene et al, 2007), which correlates with the present finding of a decrease in the level of one of the dopamine metabolites (HVA). Taken together, these findings suggest that alterations in central dopamine pathways may play a role in the induction of behavioral abnormalities in the model. Partial support for this hypothesis can be found in our demonstration that motor abnormalities were ameliorated by the D2 antagonist haloperidol.

Our study is the first to suggest the presence of autoantibodies in the sera of streptococcal-exposed rats 
and the sera of SC and PANDAS patients that react with dopamine D1 and D2 receptors. Studies are underway to determine the signaling capacity of these autoantibodies and the nature of the antibody binding site. It is also notable that we report only a small sample of well-characterized acuteonset human sera from individuals who were symptomatic with SC, or acute-onset PANDAS with characteristic small choreiform movements. These data do not reflect all of the large heterogeneous group of individuals termed PANDAS, where there are likely to be groups of patients who are less related to chorea and do not have symptoms that would associate with the autoantibody reactivity against dopamine D1 or D2 membrane receptor antigens. Indeed, subjects in a non-PANDAS group with OCD and tics have previously been shown to lack the autoantibodies seen in acute PANDAS subjects with small choreiform movements following GAS infections (Kirvan et al, 2006b).

In summary, our study links exposure to streptococcal antigen with central dopamine pathways and subsequently movement and behavior. Several pieces of evidence support our conclusion: (1) exposure to GAS antigen led to movement and behavioral changes; (2) dopamine was elevated in specific brain regions; (3) the dopamine D2 receptor antagonist haloperidol abrogated abnormal movements; and (4) elevated anti-dopamine D1 and antidopamine D2 receptor membrane antigen autoantibodies in GAS sera as well as SC and PANDAS correlated with the presence of active symptoms. The importance of our study is in the development of a model that has face validity for human symptoms, demonstrates alterations in predicted neurotransmitters in expected brain regions, and correlates with responses to pharmacotherapeutic interventions. Future research utilizing this model should provide greater understanding of how exposure to streptococcal infections or potentially other infectious agents affect the brain.

\section{ACKNOWLEDGEMENTS}

We thank Professor D Michaelson (Department of Neurobiology, Tel-Aviv University, Israel) for helpful discussions and immunohistochemical assistance, Dr Christine Kirvan (California State University at Sacramento, CA) for helpful discussions, and Reinhard Sohr (at the University Medicine Charité, Berlin, Germany) for the biochemistry analysis. We express gratitude to all of the parents who donated funds to our project. This work was funded in part by the Israel Science Foundation Grant 341/07 to DJ and ADAMS Super Center for Brain Studies at Tel Aviv University to DJ; in part by NIH-R37HL35280 (NIH MERIT AWARD) and NIH-R01HL56267 to MWC; in part by the Oklahoma Center for the Advancement of Science and Technology (OCAST) to MWC; in part by the PANDAS Research Fund at the Oklahoma University Foundation to MWC; in part by the NIMH Bench to Bedside grant to JFL, MWC, and SES; and in part by NIH-5R25MH077823 and Grifols (formerly Talecris Biotherapeutics) to JFL. The human specimens from Yale University were collected as part of the NIH-funded grants to JFL (NIH-R01MH061940, NIHP01MH049351, and NIH-K05MH076273).

\section{DISCLOSURE}

The authors declare that over the past 3 years, JFL has received compensation from NIH-5R25MH077823 and Grifols (formerly Talecris Biotherapeutics) for the conduct of an IVIG randomized clinical trial in children with Pediatric Neuropsychiatric Disorders Associated with Streptoccocal Infections (PANDAS) as part of an NIMH Bench to Bedside grant to JFL, SES, and MWC; and MWC has received compensation from NIH-R37HL35280 (NIH MERIT AWARD), NIH-R01HL56267, and Moleculera Labs, a company for diagnostics including Sydenham chorea and PANDAS.

\section{REFERENCES}

Albelda N, Joel D (2012). Animal models of obsessive-compulsive disorder: exploring pharmacology and neural substrates. Neurosci Biobehav Rev 36: 47-63.

Ayalon L, Doron R, Weiner I, Joel D (2004). Amelioration of behavioral deficits in a rat model of Huntington's disease by an excitotoxic lesion to the globus pallidus. Exp Neurol 186: 46-58.

Bhattacharyya S, Chakraborty K (2007). Glutamatergic dysfunction-newer targets for anti-obsessional drugs. Recent Pat CNS Drug Discov 2: 47-55.

Bhattacharyya S, Khanna S, Chakrabarty K, Mahadevan A, Christopher R, Shankar SK (2009). Anti-brain autoantibodies and altered excitatory neurotransmitters in obsessive-compulsive disorder. Neuropsychopharmacology 34: 2489-2496.

Brimberg L, Flaisher-Grinberg S, Schilman EA, Joel D (2007). Strain differences in 'compulsive' lever-pressing. Behav Brain Res 179: 141-151.

Bronze MS, Dale JB (1993). Epitopes of streptococcal M proteins that evoke antibodies that cross-react with human brain. J Immunol 151: 2820-2828.

Church AJ, Cardoso F, Dale RC, Lees AJ, Thompson EJ, Giovannoni G (2002). Anti-basal ganglia antibodies in acute and persistent Sydenham's chorea. Neurology 59: 227-231.

Church AJ, Dale RC, Cardoso F, Candler PM, Chapman MD, Allen ML et al (2003). CSF and serum immune parameters in Sydenham's chorea: evidence of an autoimmune syndrome? J Neuroimmunol 136: 149-153.

Colony HS, Malamud N (1956). Sydenham's chorea; a clinicopathologic study. Neurology 6: 672-676.

Conners CK, Sitarenios G, Parker JD, Epstein JN (1998). The revised Conners' Parent Rating Scale (CPRS-R): factor structure, reliability, and criterion validity. J Abnorm Child Psychol 26: 257-268.

Dale RC, Candler PM, Church AJ, Wait R, Pocock JM, Giovannoni G (2006). Neuronal surface glycolytic enzymes are autoantigen targets in post-streptococcal autoimmune CNS disease. J Neuroimmunol 172: 187-197.

Demiroren K, Yavuz H, Cam L, Oran B, Karaaslan S, Demiroren S (2007). Sydenham's chorea: a clinical follow-up of 65 patients. J Child Neurol 22: 550-554.

Dilenge ME, Shevell MI, Dinh L (1999). Restricted unilateral Sydenham's chorea: reversible contralateral striatal hypermetabolism demonstrated on single photon emission computed tomographic scanning. J Child Neurol 14: 509-513.

Fenderson PG, Fischetti VA, Cunningham MW (1989). Tropomyosin shares immunologic epitopes with group A streptococcal M proteins. J Immunol 142: 2475-2481.

Gause C, Morris C, Vernekar S, Pardo-Villamizar C, Grados MA, Singer HS (2009). Antineuronal antibodies in OCD: comparisons in children with OCD-only, OCD+chronic tics and OCD+PANDAS. J Neuroimmunol 214: 118-124.

Giedd JN, Rapoport JL, Garvey MA, Perlmutter S, Swedo SE (2000). MRI assessment of children with obsessive-compulsive disorder 
or tics associated with streptococcal infection. Am J Psychiatry 157: 281-283.

Giedd JN, Rapoport JL, Kruesi MJ, Parker C, Schapiro MB, Allen AJ et al (1995). Sydenham's chorea: magnetic resonance imaging of the basal ganglia. Neurology 45: 2199-2202.

Giedd JN, Rapoport JL, Leonard HL, Richter D, Swedo SE (1996). Case study: acute basal ganglia enlargement and obsessivecompulsive symptoms in an adolescent boy. J Am Acad Child Adolesc Psychiatry 35: 913-915.

Gordon N (2009). Sydenham's chorea, and its complications affecting the nervous system. Brain Dev 31: 11-14.

Greer JM, Capecchi MR (2002). Hoxb8 is required for normal grooming behavior in mice. Neuron 33: 23-34.

Hallett JJ, Harling-Berg CJ, Knopf PM, Stopa EG, Kiessling LS (2000). Anti-striatal antibodies in Tourette syndrome cause neuronal dysfunction. J Neuroimmunol 111: 195-202.

Hoffman KL, Hornig M, Yaddanapudi K, Jabado O, Lipkin WI (2004). A murine model for neuropsychiatric disorders associated with group A beta-hemolytic streptococcal infection. J Neurosci 24: 1780-1791.

Hogg S (1996). A review of the validity and variability of the elevated plus-maze as an animal model of anxiety. Pharmacol Biochem Behav 54: 21-30.

Homberg JR, van den Akker M, Raaso HS, Wardeh G, Binnekade $\mathrm{R}$, Schoffelmeer AN et al (2002). Enhanced motivation to selfadminister cocaine is predicted by self-grooming behaviour and relates to dopamine release in the rat medial prefrontal cortex and amygdala. Eur J Neurosci 15: 1542-1550.

Husby G, van de Rijn I, Zabriskie JB, Abdin ZH, Williams Jr RC (1976). Antibodies reacting with cytoplasm of subthalamic and caudate nuclei neurons in chorea and acute rheumatic fever. J Exp Med 144: 1094-1110.

Huyser C, Veltman DJ, de Haan E, Boer F (2009). Paediatric obsessive-compulsive disorder, a neurodevelopmental disorder? Evidence from neuroimaging. Neurosci Biobehav Rev 33: 818-830.

Joel D, Doljansky J (2003). Selective alleviation of compulsive lever-pressing in rats by $\mathrm{D} 1$, but not $\mathrm{D} 2$, blockade: possible implications for the involvement of D1 receptors in obsessivecompulsive disorder. Neuropsychopharmacology 28: 77-85.

Kirvan CA, Cox CJ, Swedo SE, Cunningham MW (2007). Tubulin is a neuronal target of autoantibodies in Sydenham's chorea. J Immunol 178: 7412-7421.

Kirvan CA, Swedo SE, Heuser JS, Cunningham MW (2003). Mimicry and autoantibody-mediated neuronal cell signaling in Sydenham chorea. Nat Med 9: 914-920.

Kirvan CA, Swedo SE, Kurahara D, Cunningham MW (2006a). Streptococcal mimicry and antibody-mediated cell signaling in the pathogenesis of Sydenham's chorea. Autoimmunity 39: 21-29.

Kirvan CA, Swedo SE, Snider LA, Cunningham MW (2006b). Antibody-mediated neuronal cell signaling in behavior and movement disorders. J Neuroimmunol 179: 173-179.

Klavir O, Flash S, Winter C, Joel D (2009). High frequency stimulation and pharmacological inactivation of the subthalamic nucleus reduces 'compulsive' lever-pressing in rats. Exp Neurol 215: 101-109.

Kurlan R, Kaplan EL (2004). The pediatric autoimmune neuropsychiatric disorders associated with streptococcal infection (PANDAS) etiology for tics and obsessive-compulsive symptoms: hypothesis or entity? Practical considerations for the clinician. Pediatrics 113: 883-886.

Kushner MG, Kim SW, Donahue C, Thuras P, Adson D, Kotlyar M et al (2007). D-cycloserine augmented exposure therapy for obsessive-compulsive disorder. Biol Psychiatry 62: 835-838.

Linthicum DS, Munoz JJ, Blaskett A (1982). Acute experimental autoimmune encephalomyelitis in mice. I. Adjuvant action of Bordetella pertussis is due to vasoactive amine sensitization and increased vascular permeability of the central nervous system. Cell Immunol 73: 299-310.
Loiselle CR, Lee O, Moran TH, Singer HS (2004). Striatal microinfusion of Tourette syndrome and PANDAS sera: failure to induce behavioral changes. Mov Disord 19: 390-396.

Marques-Dias MJ, Mercadante MT, Tucker D, Lombroso P (1997). Sydenham's chorea. Psychiatr Clin North Am 20: 809-820.

Morshed SA, Parveen S, Leckman JF, Mercadante MT, Bittencourt Kiss MH, Miguel EC et al (2001). Antibodies against neural, nuclear, cytoskeletal, and streptococcal epitopes in children and adults with Tourette's syndrome, Sydenham's chorea, and autoimmune disorders. Biol Psychiatry 50: 566-577.

Murphy TK, Husted DS, Edge PJ (2006a). Preclinical/Clinical evidence of central nervous system infectious etiology in PANDAS. Adv Neurol 99: 148-158.

Murphy TK, Sajid MW, Goodman WK (2006b). Immunology of obsessive-compulsive disorder. Psychiatr Clin North Am 29: 445-469.

Pavone P, Bianchini R, Parano E, Incorpora G, Rizzo R, Mazzone L et al (2004). Anti-brain antibodies in PANDAS versus uncomplicated streptococcal infection. Pediatr Neurol 30: 107-110.

Peterson BS, Leckman JF, Tucker D, Scahill L, Staib L, Zhang H et al (2000). Preliminary findings of antistreptococcal antibody titers and basal ganglia volumes in tic, obsessive-compulsive, and attention deficit/hyperactivity disorders. Arch Gen Psychiatry 57: 364-372.

Piontkewitz Y, Arad M, Weiner I (2010). Risperidone administered during asymptomatic period of adolescence prevents the emergence of brain structural pathology and behavioral abnormalities in an animal model of schizophrenia. Schizophr Bull 37: $1257-1269$.

Quinn A, Kosanke S, Fischetti VA, Factor SM, Cunningham MW (2001). Induction of autoimmune valvular heart disease by recombinant streptococcal m protein. Infect Immun 69: 4072-4078.

Rosenberg DR, Mirza Y, Russell A, Tang J, Smith JM, Banerjee SP et al (2004). Reduced anterior cingulate glutamatergic concentrations in childhood OCD and major depression versus healthy controls. J Am Acad Child Adolesc Psychiatry 43: 1146-1153.

Shannon KM, Fenichel GM (1990). Pimozide treatment of Sydenham's chorea. Neurology 40: 186.

Singer HS, Loiselle C (2003). PANDAS: a commentary. J Psychosom Res 55: 31-39.

Singer HS, Mink JW, Loiselle CR, Burke KA, Ruchkina I, Morshed $S$ et al (2005). Microinfusion of antineuronal antibodies into rodent striatum: failure to differentiate between elevated and low titers. J Neuroimmunol 163: 8-14.

Singer HS, Minzer K (2003). Neurobiology of Tourette's syndrome: concepts of neuroanatomic localization and neurochemical abnormalities. Brain Dev 25(Suppl 1): S70-S84.

Singer HS, Morris C, Grados M (2009). Glutamatergic modulatory therapy for Tourette syndrome. Med Hypotheses 74: 862-867.

Snider LA, Swedo SE (2004). PANDAS: current status and directions for research. Mol Psychiatry 9: 900-907.

Stein DJ (2002). Obsessive-compulsive disorder. Lancet 360: 397-405. Stollerman GH (1997). Rheumatic fever. Lancet 349: 935-942.

Swedo SE, Leonard HL, Garvey M, Mittleman B, Allen AJ, Perlmutter S et al (1998). Pediatric autoimmune neuropsychiatric disorders associated with streptococcal infections: clinical description of the first 50 cases. Am J Psychiatry 155: 264-271.

Swedo SE, Leonard HL, Rapoport JL (2004). The pediatric autoimmune neuropsychiatric disorders associated with streptococcal infection (PANDAS) subgroup: separating fact from fiction. Pediatrics 113: 907-911.

Swedo SE, Rapoport JL, Cheslow DL, Leonard HL, Ayoub EM, Hosier DM et al (1989). High prevalence of obsessivecompulsive symptoms in patients with Sydenham's chorea. Am J Psychiatry 146: 246-249.

Taylor JR, Morshed SA, Parveen S, Mercadante MT, Scahill L, Peterson BS et al (2002). An animal model of Tourette's syndrome. Am J Psychiatry 159: 657-660. 
Umene W, Yoshimura R, Hori H, Nakano H, Sugita A, Shimono M et al (2007). Blood levels of catecholamine metabolites and brain-derived neurotrophic factor in a case of Sydenham's chorea. World J Biol Psychiatry 10: 248-251.

Urakawa S, Hida H, Masuda T, Misumi S, Kim TS, Nishino H (2007). Environmental enrichment brings a beneficial effect on beam walking and enhances the migration of doublecortinpositive cells following striatal lesions in rats. Neuroscience 144: 920-933.
Weindl A, Kuwert T, Leenders KL, Poremba M, Grafin von Einsiedel H, Antonini A et al (1993). Increased striatal glucose consumption in Sydenham's chorea. Mov Disord 8: 437-444.

Yaddanapudi K, Hornig M, Serge R, De Miranda J, Baghban A, Villar G et al (2009). Passive transfer of streptococcus-induced antibodies reproduces behavioral disturbances in a mouse model of pediatric autoimmune neuropsychiatric disorders associated with streptococcal infection. Mol Psychiatry 15: 712-726.

Supplementary Information accompanies the paper on the Neuropsychopharmacology website (http://www.nature.com/npp) 